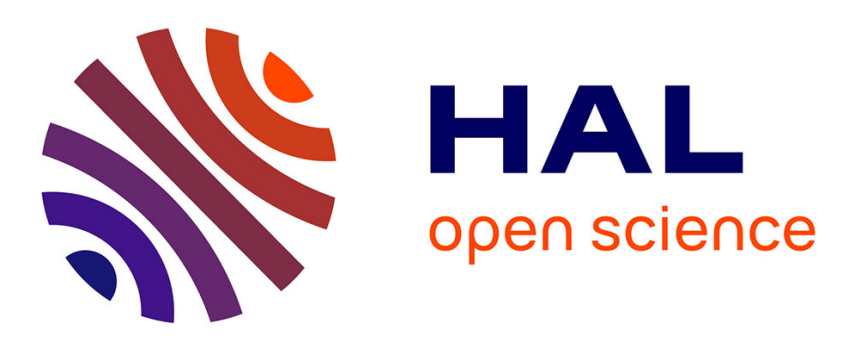

\title{
The Birth of Artificial Intelligence: First Conference on Artificial Intelligence in Paris in 1951?
}

\author{
Herbert Bruderer
}

\section{To cite this version:}

Herbert Bruderer. The Birth of Artificial Intelligence: First Conference on Artificial Intelligence in Paris in 1951?. IFIP International Conference on the History of Computing (HC), May 2016, Brooklyn, NY, United States. pp.181-185, 10.1007/978-3-319-49463-0_12 . hal-01620145

\section{HAL Id: hal-01620145 \\ https://hal.inria.fr/hal-01620145}

Submitted on 20 Oct 2017

HAL is a multi-disciplinary open access archive for the deposit and dissemination of scientific research documents, whether they are published or not. The documents may come from teaching and research institutions in France or abroad, or from public or private research centers.
L'archive ouverte pluridisciplinaire HAL, est destinée au dépôt et à la diffusion de documents scientifiques de niveau recherche, publiés ou non, émanant des établissements d'enseignement et de recherche français ou étrangers, des laboratoires publics ou privés. 


\title{
The Birth of Artificial Intelligence: First Conference on Artificial Intelligence in Paris in 1951?
}

\author{
Herbert Bruderer \\ ETH Zurich, Switzerland \\ (bruderereretired.ethz.ch; herbert.bruderer@bluewin.ch)
}

\begin{abstract}
The 1956 Dartmouth conference is often considered as the cradle of artificial intelligence. There is a controversy on its origin. Some historians of computing believe that Turing or Zuse were the fathers of machine intelligence. However, the first working chess-playing automaton was developed by Torres Quevedo by 1912. Moreover, there was a large and important (but forgotten) European conference on computing and human thinking in Paris in 1951.
\end{abstract}

Keywords: Paris conference on artificial intelligence, Spanish chess automaton by Torres Quevedo, Turing's impact

\section{Paris International Computer Conference}

There were three conferences on calculating machines in Britain in 1949 (University of Cambridge), 1951 (University of Manchester) and 1953 (National Physical Laboratory, London). However, the most important early European computer conference took place in Paris in 1951. This international congress organized by the Centre National de la Recherche Scientifique (CNRS) is almost forgotten despite its famous participants. The 35 papers were translated into French which seems to be quite unique. The reason why this international meeting is nearly unknown is probably the fact that the voluminous proceedings (589 pages) were published only in French, they do not contain English essays. The gathering combined computing machines and human thinking. Its meaningful title was «Les machines à calculer et la pensée humaine.».

\section{Famous Participants from Europe and USA}

Among the participants from ten countries were:

- Aiken (Harvard)

- Ashby (Gloucester)

- Booth (London)

- Bowden (Manchester) 
- Colebrook (London)

- Couffignal (Paris)

- Hartree (Cambridge)

- Kilburn (Manchester)

- McCulloch (Chicago)

- Picone (Rom)

- Stiefel (Zurich)

- Torres Quevedo (Madrid)

- Uttley (London)

- van Wijngaarden (Amsterdam)

- Walter (Bristol)

- Walther (Darmstadt)

- Wiener (Cambridge, Mass.)

- Wilkes (Cambridge)

- Williams (Manchester)

- Womersley (Letchworth).

\section{Demonstration of Several Automata}

There were several demonstrations: automatic chess player, Telekine and analog calculator, all operated by Leonardo Torres Quevedo's son Gonzales, artificial animals by W. Grey Walter, Homeostat by W. Ross Ashby.

Both the chess automata still exist in Madrid. The Austrian pioneer Heinz Zemanek (transistorized computer Mailüfterl) who died in 2015 played against the second version at the world fair of Brussels in 1958.

\section{Papers on Computers and Human Brain}

Many pioneers presented their relay and electronic computers, for example: Howard Aiken (Harvard University): Mark IV, Maurice Wilkes (University of Cambridge): EDSAC, Frederic Williams (University of Manchester): Manchester Mark, Francis Morley Colebrook (National Physical Laboratory, London): ACE. Göran Kjellberg (Sweden) and Eduard Stiefel (Switzerland) reported on the first continental European computers (Bark and Zuse Z4).

Paul Chauchard, Louis Couffignal, L. Delpech, A. Fessard, Henri Gastaut, Warren S. McCulloch/Walter H. Pitts, W. Grey Walter, and Norbert Wiener lectured on the relations between the machine and the human brain (for details see references). 


\section{$5 \quad$ Turing's Revolution?}

It is remarkable that Alan Turing did not attend the Paris conference. At this time he was living in Manchester (after leaving the ACE project in London). There is a new book entitled "Turing's revolution" (Springer 2015). In 1951, 15 years after his 1936 paper on the universal Turing machine, Turing's work was almost unknown. His impact on the building of the early stored-program computers was rather small. In a leading European book on program controlled electronic digital computers (Rutishauser, Heinz; Speiser, Ambros Paul; Stiefel, Eduard: Programmgesteuerte digitale Rechengeräte (elektronische Rechenmaschinen), Birkhäuser Verlag, Basel 1951) Turing's universal machine is not even mentioned.

Arnold A. Cohen writes in his introduction to a significant historical book: "Although the ERA book may have been the only one of its kind published in America, it was not alone in the world at the time. Rutishauser, Speiser, and Stiefel's comprehensive review "Program controlled digital computing devices (Electronic computing machines)", appeared in four installments (1950-1951) in the Swiss journal Zeitschrift für angewandte Mathematik und Physik. This excellent tutorial, with its extensive bibliography, was not widely available in the U.S." (see Engineering Research Associates, Inc.: High-speed computing devices, Tomash publishers, Los Angeles, San Francisco 1983, page XIX).

At the Paris conference, Francis Morley referred to Turing's abstract universal machine but he did not recognize a connection between the stored-program concept and modern electronic digital computers. Obviously, there was no Turing revolution concerning the construction of stored-program computers.

\section{Conclusions}

Was the Dartmouth Summer Research Project on Artificial Intelligence (John McCarthy, Marvin L. Minsky, Nathaniel Rochester, Claude E. Shannon) which took place in 1956 at Dartmouth College in Hanover, New Hampshire the first major event for AI? This seems rather doubtful. The Paris congress, sponsored by the Rockefeller Foundation, included several contributions on digital and human computers.

Acknowledgment. The author is grateful for discussions with Jürgen Schmidhuber. 


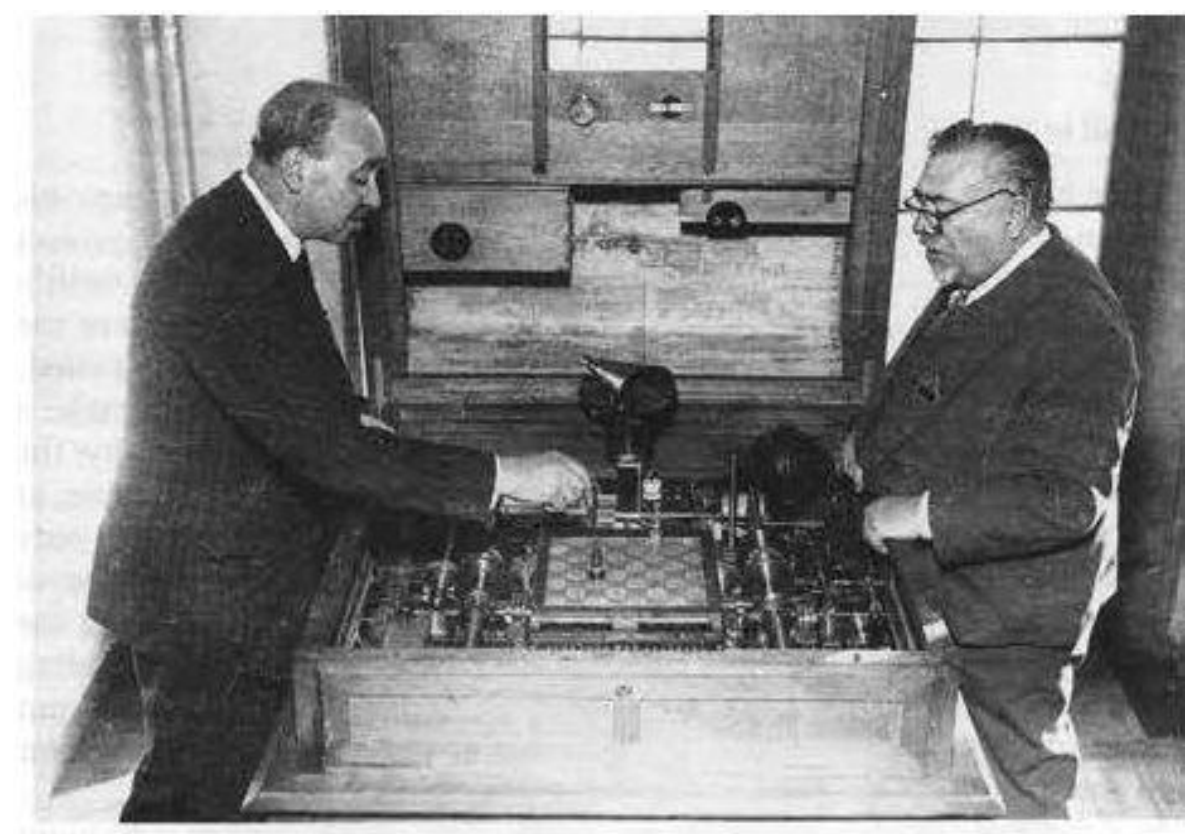

Norbert Wiener playing the chess automaton (designed by Torres y Quevedo) at the 1951 Cybernetics Conference in Paris

Fig.1. The Spanish engineer Gonzales Torres Quevedo presented his father's chess machine $E l$ ajedrecista at the Paris conference on computers and human thinking. Norbert Wien played on 12 or 13 January 1951 against the automaton (picture from Vernon Pratt: Thinking machines. The evolution of artificial intelligence, Basil Blackwell Ltd, Oxford 1987, page 191, original source unknown).

\section{References}

1 Bruderer, H.: Meilensteine der Rechentechnik. Zur Geschichte der Mathematik und der Informatik (Milestones in Analog and Digital Computing. Contributions to the History of Mathematics and Information Technology), de Gruyter, Berlin/Boston 2015, 850 pages

2 Bruderer, H.: Computing History outside UK and USA: some selected Landmarks from Continental Europe, in: Communications of the ACM (forthcoming)

3 Chauchard, P.: La commande centrale de la machine nerveuse, in: Joseph Pérès (ed.): Les machines à calculer et la pensée humaine, Paris, 8-13 janvier 1951, Colloques internationaux du Centre national de la recherche scientifique, Nr. 37, Editions du Centre national de la recherche scientifique (CNRS), Paris 1953, pages 531-536

4 Couffignal, L.: Quelques analogiques nouvelles entre structures de machines à calculer et structures cérébrales, in: Joseph Pérès (ed.): Les machines à calculer et la pensée humaine, Paris, 8-13 janvier 1951, Colloques internationaux du Centre national de la recherche 
scientifique, Nr. 37, Editions du Centre national de la recherche scientifique (CNRS), Paris 1953, pages $549-562$

5 Delpech, L.: Perspectives psychologiques et machines à penser, in: Joseph Pérès (ed.): Les machines à calculer et la pensée humaine, Paris, 8-13 janvier 1951, Colloques internationaux du Centre national de la recherche scientifique, Nr. 37, Editions du Centre national de la recherche scientifique (CNRS), Paris 1953, pages 539-548

6 Fessard, A.: Sur un „défaut“ propre à la machine nerveuse, in: Joseph Pérès (ed.): Les machines à calculer et la pensée humaine, Paris, 8-13 janvier 1951, Colloques internationaux du Centre national de la recherche scientifique, Nr. 37, Editions du Centre national de la recherche scientifique (CNRS), Paris 1953, pages 517-528

7 Gastaut, H.: Les machines à calculer et le cerveau humain, in: Joseph Pérès (ed.): Les machines à calculer et la pensée humaine, Paris, 8-13 janvier 1951, Colloques internationaux du Centre national de la recherche scientifique, Nr. 37, Editions du Centre national de la recherche scientifique (CNRS), Paris 1953, pages 447-459

8 Mc Culloch, W. S.; Lettvin, J. Y.; Pitts, W. H.; Dell, P. C.: Une comparaison entre les machines à calculer et le cerveau, in: Joseph Pérès (ed.): Les machines à calculer et la pensée humaine, Paris, 8-13 janvier 1951, Colloques internationaux du Centre national de la recherche scientifique, Nr. 37, Editions du Centre national de la recherche scientifique (CNRS), Paris 1953, pages 425-443

9 Pérès, J. (ed.): Les machines à calculer et la pensée humaine, Paris, 8-13 janvier 1951, Colloques internationaux du Centre national de la recherche scientifique, Nr. 37, Editions du Centre national de la recherche scientifique (CNRS), Paris 1953, XIX, 570 pages

10 Rutishauser, H.; Speiser, A. P.; Stiefel, E.: Programmgesteuerte digitale Rechengeräte (elektronische Rechenmaschinen), Birkhäuser Verlag, Basel 1951, 102 pages

11 Torres-Quevedo, G.: Les travaux de l'École espagnole sur l'automatisme, in: Joseph Pérès (ed.): Les machines à calculer et la pensée humaine, Paris, 8-13 janvier 1951, Colloques internationaux du Centre national de la recherche scientifique, Nr. 37, Editions du Centre national de la recherche scientifique (CNRS), Paris 1953, pages 361-381

12 Torres-Quevedo, G.: Présentation des appareils de Leonardo Torres-Quevedo, in: Joseph Pérès (ed.): Les machines à calculer et la pensée humaine, Paris, 8-13 janvier 1951, Colloques internationaux du Centre national de la recherche scientifique, Nr. 37, Editions du Centre national de la recherche scientifique (CNRS), Paris 1953, pages 383-406

13 Walter, W. G.: Réalisation mécanique de modèles de structure cérébrale, in: Joseph Pérès (ed.): Les machines à calculer et la pensée humaine, Paris, 8-13 janvier 1951, Colloques internationaux du Centre national de la recherche scientifique, Nr. 37, Editions du Centre national de la recherche scientifique (CNRS), Paris 1953, pages 407-420

14 Wiener, N.: Les machines à calculer et la forme (Gestalt), in: Joseph Pérès (ed.): Les machines à calculer et la pensée humaine, Paris, 8-13 janvier 1951, Colloques internationaux du Centre national de la recherche scientifique, Nr. 37, Editions du Centre national de la recherche scientifique (CNRS), Paris 1953, pages 461-463

15 Zemanek, H.: Central European prehistory of computing, in: Nicholas Constantine Metropolis; Jack Howlett; Gian-Carlo Rota (eds.): A history of computing in the twentieth century. A collection of essays, Academic press, New York, London 1980, pages 587-609. 